\title{
Towards Improving Skin Cancer Detection Using Transfer Learning
}

\author{
S. Sasikala ${ }^{1 *}$, S. Arun Kumar ${ }^{2}$, S.N. Shivappriya ${ }^{3}$ and Priyadharshini $T^{4}$ \\ ${ }^{1,3}$ Assosciate Professor, ${ }^{2}$ Assistant Professor, ${ }^{4} U G$ Scholar \\ Department of Electronics and Communication Engineering, \\ Kumaraguru College of Technology, Coimbatore-49, India
}

\section{ABSTRACT}

In present time, skin cancer is the deadliest disease among humans. In US, two persons die every hour owing to skin cancer. Skin cancer is developed on the body when exposed to sunlight and is the abnormal growth of the skin cell. The patient's life can be saved through earlier and faster detection of skin cancer. The formal method of skin cancer detection is Biopsy, it is done by removing the skin cells and testing the samples in a clinical lab. Biopsy method is invasive and time-consuming. With the newer technologies, early detection of skin cancer at the initial stage is possible. Image processing techniques are instrumental in the health care industry to detect abnormalities in the human body. In this work, Convolutional Neural Network (CNN) algorithm with four different transfer learning techniques are used to classify the images of the skin with dermoscopic analysis which enables fast detection. A CNN model is trained using a dataset of 3700 clinical images and its performance is tested over 660 images which represent the identification of deadliest skin cancer. A considerable improvement in accuracy of skin cancer detection using deep learning architecture ResNet34 provides a reliable approach for early detection and treatment.

KEY WORDS: CONVOLUTIONAL NEURAL NETWORK, DEEP LEARNING, DETECTION, TRANSFER LEARNING, SKIN CANCER.

\section{INTRODUCTION}

Skin cancer is the uncontrollable growth of damaged cells in the outer most layer of the skin. This is because of damage in DNA sequence due to the environmental factors like cigarette smoke and exposure to Ultra Violet (UV) light. DNA damage triggers mutation which leads to rapid multiplication of skin cells that forms malignant tumors [Miller .et. al. (1994)].

\section{ARTICLE INFORMATION}

*Corresponding Author: sasikala.s.ece@kct.a.in

Received 05th Oct 2020 Accepted after revision 10th Dec 2020

Print ISSN: 0974-6455 Online ISSN: 2321-4007 CODEN: BBRCBA

Thomson Reuters ISI Web of Science Clarivate Analytics USA and Crossref Indexed Journal

\section{Clarivate
Analytics}

NAAS Journal Score 2020 (4.31) SJIF: 2020 (7.728)

A Society of Science and Nature Publication,

Bhopal India 2020. All rights reserved.

Online Contents Available at: http//www.bbrc.in/

Doi: http://dx.doi.org/10.21786/bbrc/13.11/13
Skin cancer is classified into Melanoma, Basal Cell Carcinoma (BCC) and Squamous Cell Carcinoma (SCC). Melanoma is the most dangerous type of cancer which leads to death that usually appears on the moles and the areas on the skin which is exposed to sunlight as well as not exposed to sunlight. The affected part of the skin contains melanocytes that spread to other parts of the body. BCC is the most laggard growing and never be large in size. It appears on the skin exposed areas such as hand, face, leg, ears and scalp. It usually matures as an ulcer and does not improve. The early detection of this can be curable. Some are hostile and cannot be treated because it spreads to the deeper cells of the tissue. SQC appears on the sun exposed part and on the incurable inflammation part of the body and occurs in the person who has low immune power. It is large, appears in incurable scars and in lips. The early detection is possible. 
Benign is a non-cancerous which does not spread to any other parts of the body. It is caused due to exposure of sunlight, inflammation of skin, infections, and genetics.

Melanoma mostly occurs in the skin rarely in the mouth and intestines with the abnormal cells that contain melanocytes which control the pigment in our skin. For women, melanoma mostly occurs on the legs and for men on the back. They usually develop from the mole with abnormal changes as an increase in size, changes in the color, causes itches or skin breakdown. It can occur in the areas between fingernails, palms, toenails and eyes [Miller .et. al. (1994)]. Benign usually appears on the skin which is highly exposed to sunlight such as face, shoulders, neck, hand and leg. This appears as lump and looks like patches which continues after a week and develops over a month or a year.

Skin cancer is the common type of cancer in worldwide and especially in US. By the age of 70, skin cancer will be developed by 1 in 5 Americans. In every hour, more than 2 people die because of skin cancer. Risk for melanoma will be doubled while exposure of sunburns is more than 5 in number. Early detection helps to survive for 5 years and the survival rate is 99 percent. At least $40 \%$ of cases have skin cancer when globally accounting for common cancer. Non-melanoma skin cancer occurs 2 to 3 million people per year. Globally in 2012, 232,000 people were in skin cancer, and 55,000 people died. According to the survey of last 20 to 40 years, Australia (white people), New Zealand and South Africa People have the highest rate of Skin cancer in the world [Apalla, Z.et. al. (2017)].

The early detection involves Biopsy method, in that the damaged skin is removed and tested in laboratories which take longer duration for the detection of skin cancer and it is more painful method. Computer Aided Diagnosis (CAD) is used to avoid the longer duration consumption and it is a non-invasive method. Many works in literature [Sasikala, S. et al. (2018) \&t (2020)] have focused on machine learning, feature transformation, optimisation, and deep learning for improving the cancer diagnosis. Henceforth, the proposed work aims to develop a CAD system for early identification of skin cancer using deep learning.

The significant contributions of the proposed work are:

- To design a cost-effective CAD system for the early identification of skin cancer.

- To construct a deep learning model that can detect and categorize given image into either a benign type or malignant type.

- $\quad$ To reduce training time by using pre-trained model with high accuracy rate.

Related Works: Automatic detection of skin cancer involved pre-processing and the post-processing techniques for the classification of the image with high accuracy. The Pre-processing increases the performance by noise removal. Post-processing enhanced the image quality and the boundary of the cancer cell was enhanced. The problem is that the dataset was too small. There was no uniqueness in the image processing technique and the variations between the dermoscopy and the digital image were large. By increasing the number of images in the data set, high accuracy over training and testing data set could be obtained [Lau, H.T. and Al-Jumaily, A., (2009)].

Skin cancer is the most common disease in human and its incidence is increasing dramatically. The newer technology-based detecting skin cancer is recommended for accurate identification but the percentage of detection by computer is comparatively high with manual detection. Here Digital Dermoscopy is widely considered as one of the most effective means to classify the skin cancers. Segmentation of images is done using K-means algorithm. It includes various stages like skin image, enhancement, lesion segmentation, feature extraction and finally classifying it to normal and abnormal. Convolution Neural Network (CNN) for detection of images, which is much cost-efficient in comparison with digital dermoscopy gives accurate output with appropriate detection of the cancer. The model proposed the automatic method of detecting skin cancer from the photographed image which was captured from the affected area of the skin. In this, Support Vector Machine (SVM) algorithm is used to classify the image which was either melanoma or benign. A total of 100 image for melanoma and 100 for benign was used in the study. The eminent drawback of this method is that the data set did not contain the dark-skinned images. To overcome these difficulties, more images are used to train the data and to test the trained data with all types of images of the skin [Esteva, A et. al (2017)].

Three methods were used for the segmentation of Otsu's method, gradient vector flow and color-based segmentation using K-mean clustering. Otsu's method did not require any variation in the parameter for the different skin lesions. The gradient vector flow had the active contour to boundary concavities although with the presence of the noises. The drawback of it was the execution speed to converge to the object. This method required the changing parameter for the different skin lesions. In color-based classification, it had the possibility of reducing the computational cost calculation for every pixel in the image. To overcome these drawbacks, the Convolutional Neural Network (CNN) algorithm with the back-propagation model is used for the fast execution to converge the object image for training [Rubegni $\mathrm{P}$ et al. (2012)].

An artificial neural network algorithm was used for the classification of the image and feature extraction for the thresholding. This method involved a binary classifier for classifying benign and malignant tumor. By the result of the artificial neural network, the accuracy was low. The different types of classification methods and image 
processing techniques could be used for high accuracy and the accurate detection of the cancer [Hosny, K.M., Kassem, M.A. and Foaud, M.M. (2018)].

Melanoma and benign lesions have high similarity, due to this it takes a long time to identify and classify. The automatic classification of skin lesions helps to reduce time, efforts and one of the best ways to give an accurate identification of lesions. The use of transfer learning and pre-trained neural network has been experimented. In this method, binary classifier model was used. This proposed method provides accuracy around 96 percentage. The weights are fine-tuned and the dataset undergoes various rotation angles to overcome problems. From this paper, we included a feature for greater accuracy for the better identification of lesions [Mahmoud, M.K.A., Al-Jumaily, A. and Takruri, M (2011)].

Rule based approach, back propagation and neural network was used to select the features and to classify the lesions. The lesions were either melanoma or benign. As in this, the number of correct classifications increased. The neural network handled the complex relations among the identification of lesions. This model had a drawback of slow convergence rate and the trapping of the local minima. To overcome this, CNN could be used to increase the convergence rate and reduce the complexity of classifying the images to increase the trapping of the local minima [Mendes, D.B. and da Silva, N.C. (2018)]. ResNet-134 architecture was used that was trained over 3797 images and later 956 images were tested with the network and achieved an accuracy of about $78 \%$. This technique took a long time to train the data set of approximately 35 hours. The images were not trained properly and led to the wrong prediction of the cancer lesions and made a problem for humans. By using ResNet 34, the time consumption to train the data set and the accuracy could be reduced [Jain, S. and Pise, N. (2015)].

SVM algorithm and snake active contour were used for image segmentation. To reduce the complexity in SVM, the snake parameters were used to predict the initial curve. The segmentation and the classification of the images are not accurate. To overcome these difficulties, CNN with ResNet 34 architecture could be used to detect the image accurately without any discrepancy [Aswin, R.B., Jaleel, J.A. and Salim, S. (2014)]. Computer-aided approach was used to detect skin cancer. The steps involved in the detection of cancer are image pre-processing, segmentation, feature extraction, and classification. Image resizing affected the quality of classification. By using this method, segmentation process involved the drawback was that the large stacks of data set could not be used for the classification of the image. To overcome these drawbacks, the CNN algorithm is used to classify large data set for accurate results [Hosny, K.M., Kassem, M.A. and Foaud, M.M.(2019)].

Transfer learning was applied with AlexNet by replacing last layer to classify the different types of lesions as melanoma and benign by softmax classification. CNN algorithm was used in this method to classify the images as melanoma or benign. The performance accuracy of the proposed model was high. The back-propagation method was used to fine-tune the weights to classify the images accurately. From the literature, it is observed that using CNN with the ResNet 34 architecture gives high accuracy with the low error rate with the probability of the classifier. Therefore, the proposed method for classifying benign and malignant tumors using CNN with ResNet architecture.

\section{MATERIAL AND METHODS}

In the present time, machine learning and deep learning approaches are used in healthcare for improved diagnosis. In this work, Convolutional Neural Network (CNN) algorithm with four different transfer learning approaches viz, AlexNet, VGG16, ResNet50, ResNet34 are used to classify the images of the skin with dermoscopic analysis which enables fast detection. The workflow with RESNET 34 transfer learning is proposed in figure 1.

Figure 1: Architecture for Skin Cancer detection

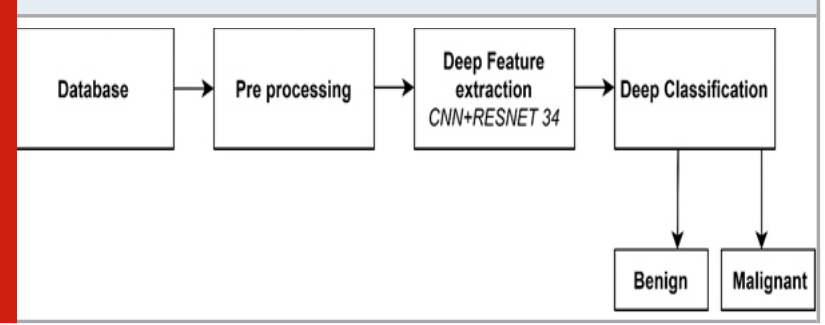

Table I represents the number of layers and the parameters used in the four transfer learning approaches used in this work.

Table 1. Different types of CNN Architectures Used

\begin{tabular}{|l|c|c|}
\hline $\begin{array}{l}\text { Name of the } \\
\text { Architecture }\end{array}$ & $\begin{array}{c}\text { Number } \\
\text { of Layers }\end{array}$ & $\begin{array}{c}\text { Number of } \\
\text { Parameters }\end{array}$ \\
\hline Alexnet & 8 & $61 \mathrm{M}$ \\
\hline VGG 16 & 16 & $138 \mathrm{M}$ \\
\hline ResNet 34 & 34 & $21.282 \mathrm{M}$ \\
\hline ResNet 50 & 50 & $23.521 \mathrm{M}$ \\
\hline
\end{tabular}

Figure 2: Benign tumour Malignant tumour

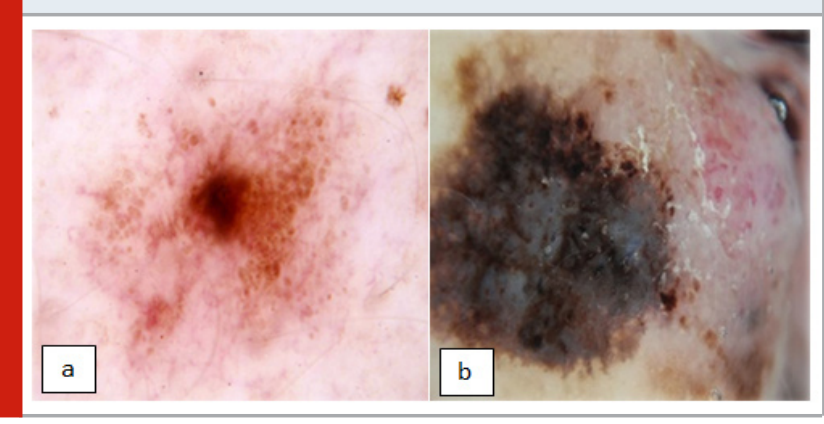


Data Base: The data sets are clinical images which are collected from Kaggle [kaggle.com]. The dataset has images of benign and malignant skin lesions in a balanced number. It consists of malignant and benign classes with 1800 images each. 70\% of data is used for training and 30\% for testing. A sample image in database is shown in figure 2.

Transfer learning is one of the machine learning techniques, used to develop a model for any recognition/ classification task. Also, it is the recommended method in deep learning where pre-trained models are used as the starting point of analysis. In transfer learning, a model trained on a single task is repurposed on another related task. It is an optimization that allows quick progress when modelling the new task.

Convolutional Neural Network (CNN): A Convolutional Neural Network (CNN or ConvNet) is a specialized type of artificial neural network (ANN) which is used in image processing, recognition and this is designed to process pixel data. CNN has some layers as the input layer, an output layer and hidden layers (multiple convolutional layers, pooling layers, fully connected layers and normalization layers) [Krizhevsky, A., Sutskever, I. and Hinton, G.E(2017)]. The figure 3 represents the highly performing architecture of CNN. Input Layer. The input layer or volume is an image and the dimensions of the image are [width $\mathrm{x}$ height $\mathrm{x}$ depth]. This dimension denotes the matrix of pixel values. For example, input is [32x32x3]. So width=32, height=32 and depth=3. Here the depth represents R, G, B channels. And the input volume should be divisible number of times by 2 .

Figure 3: Convolutional Neural Network with its layers

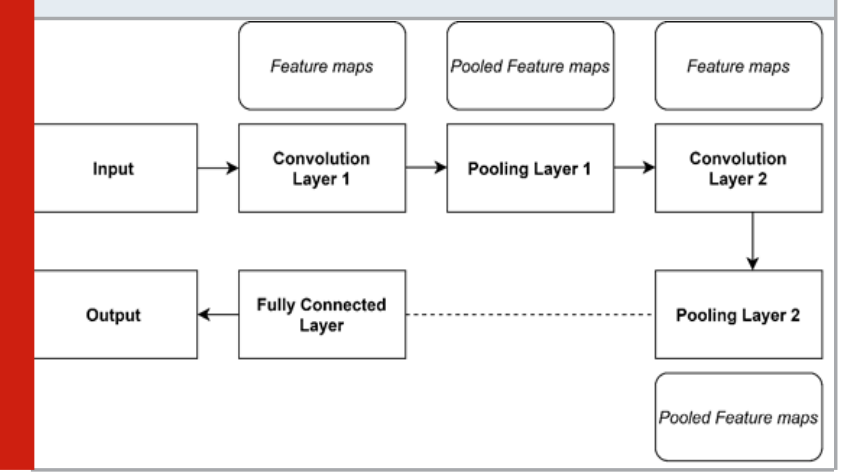

Convolutional Layer: The main objective of this layer is feature extraction from the input layer. A small part of the image is joined to the convolutional layer to reduce the expense. For this purpose, dot products are applied between a filter and a receptive field on all the dimensions. After this dot product, the output volume with a single integer is obtained. This is known as a feature map. This process is done for the entire input image. The input for the next layer will be the output of the present layer. For feature detection, filter, kernel or feature detector which is a small matrix is used. The size of the typical filter on the first layer of $\mathrm{CNN}$ is [5x5x3]. After computing the dot product and by sliding the filter over the image, a convolved feature, activation map or feature map is formed (output volume). The number of filters is known as depth. The size of the filter and the receptive field, which is the local region of the input volume are the same. The set of neurons that are all pointing to the receptive field is known as depth column or fiber. Stride is used for spatially producing smaller output volumes.

Pool Layer: The function of this layer reduces the computational complexity of the model and spatial dimensions of the given input data. Over fitting is also controlled by this layer. It does not depend on the depth slice of the input. Different functions are Max pool, Average pool or L2 norm-pool. Max pooling is the most important part of the input layer.

Table 2. Results obtained with four CNN models

\begin{tabular}{|l|c|c|c|c|}
\hline $\begin{array}{l}\text { MODEL/ } \\
\text { Metrics }\end{array}$ & ResNet34 & ResNet50 & VGG16 & AlexNet \\
\hline Accuracy & $88.4 \%$ & $76.9 \%$ & $69.2 \%$ & $65.3 \%$ \\
\hline Error Rate & $17.6 \%$ & $30.7 \%$ & $26.9 \%$ & $26.9 \%$ \\
\hline Sensitivity & 0.875 & 0.875 & 0.875 & 1 \\
\hline Specificity & 0.778 & 0.875 & 0.556 & 0.5 \\
\hline F1 score & 0.736 & 0.736 & 0.608 & 0.64 \\
\hline
\end{tabular}

Table 3. Epoch Vs accuracy and error rate table

\begin{tabular}{|l|c|c|c|c|}
\hline EPOCH & $\begin{array}{c}\text { TRAINING } \\
\text { LOSS }\end{array}$ & $\begin{array}{c}\text { VALID } \\
\text { LOSS }\end{array}$ & $\begin{array}{c}\text { ACCURACY } \\
(\%)\end{array}$ & $\begin{array}{c}\text { ERROR } \\
\text { RATE }\end{array}$ \\
\hline 0 & 0.8663 & 0.5069 & 79.78 & 0.202 \\
\hline 10 & 0.4237 & 0.3699 & 84.49 & 0.1550 \\
\hline 20 & 0.2985 & 0.3901 & 81.76 & 0.1823 \\
\hline 30 & 0.2321 & 0.3265 & 86.77 & 0.1322 \\
\hline 40 & 0.1562 & 0.3912 & 86.02 & 0.1398 \\
\hline 50 & 0.1219 & 0.3808 & 87.84 & 0.1215 \\
\hline 60 & 0.1120 & 0.3591 & 88.14 & 0.1185 \\
\hline 70 & 0.084 & 0.3691 & 88.45 & 0.1155 \\
\hline 80 & 0.0655 & 0.3957 & 88.90 & 0.1109 \\
\hline 90 & 0.0486 & 0.3945 & 88.75 & 0.1124 \\
\hline 99 & 0.0661 & 0.3858 & 88.6 & 0.1139 \\
\hline
\end{tabular}

Fully Connected Layer: The main function of this layer is to connect each neuron of one layer to each neuron in another layer. This layer uses the softmax activation function. This function is used for classifying the generated features of the input images into various classes. This classification is based on the training dataset. 
Resnet: ResNet is the short form for a residual network which is an ANN kind. ResNet could train extraordinary deep neural networks with more than 500 layers and still achieves powerful performance. According to the number of layers in the network, the number of parameters is selected. At its core, it uses batch normalization. The input layer is adjusted by batch normalization for increasing the performance of the network. The problem of covariate shift is reduced. The identity connection helps to preserve the network from vanishing gradient problem. This connection is used in ResNet. Bottle design residual network is used by Deep Residual Network to increase the network performance [He, K., Zhang, X., Ren, S. and Sun, J. (2016)]. Res-Net converges faster than plain counterpart. It also reduces degradation problem. ResNet-34 architecture offers bigger batch size which reduces training time. Further, ResNet-34 has a better validation error of 5.6\% when compared to VGG. This is due to different depths of models. Hence Resnet is used in the proposed work.

\section{RESLTS AND DISCUSSION}

The data sets are processed with pre-trained CNN ResNet 34 networks to classify the type of skin cancer that is either benign or malignant. The final testing stage is done by choosing random skin lesion image. It is then tested for accuracy and error rate. Skin cancer detection is done with python for Transfer learning. The initial results obtained with all four approaches are tabulated in the Table 2 below. Among the four models RESNET 34 shows better performance than the other three models.

ResNet 34 alters only the first and last layer that is classification layers for more efficiency when compared with already proposed methods. By using a pre-trained RESNET model and GPU, the training time had been reduced to 1 hour. The detection of Skin cancer involves binary classifier as malignant and benign. Skin cancer detection is done with a different number of Epochs and learning rates. The lower number of epoch and a higher learning rate results in more error probability and less accuracy. The number of epochs given and corresponding output are listed in the Table 3 for training ResNet using higher Learning Rate (LR). The loss vs accuracy obtained by initially testing with 100 images of data sets with 70 for train and validation respectively is studied. This resulted in high validation loss and less accuracy (62\%). To improve the system accuracy the training process used several data sets. Further training with 4360 images (Train - 3700, Valid - 660) of data set gives improved accuracy of about $88.6 \%$ in 100 epochs.

For the accurate detection of skin cancer, the learning rate is given as lower and higher as 1e-06 and 1e-03 with a loss of 0.06 and 0.145 respectively. With the maximum learning rate of 1e-03, the following accuracy and error rate for 10 epochs are identified. This gives the maximum accuracy of $90.12 \%$ and the minimum error rate of $9.8 \%$ after 10 epochs. The table below shows the change in accuracy and error rate for every epoch. The results are tabulated in Table IV.

\begin{tabular}{|c|c|c|c|c|}
\hline EPOCH & $\begin{array}{l}\text { TRAINING } \\
\text { LOSS }\end{array}$ & $\begin{array}{l}\text { VALID } \\
\text { LOSS }\end{array}$ & $\begin{array}{c}\text { ACCURACY } \\
(\%)\end{array}$ & $\begin{array}{l}\text { ERROR } \\
\text { RATE }\end{array}$ \\
\hline 0 & 0.8663 & 0.3291 & 89.51 & 0.1048 \\
\hline 1 & 0.4237 & 0.3354 & 89.96 & 0.1003 \\
\hline 2 & 0.2985 & 0.3285 & 89.81 & 0.1018 \\
\hline 3 & 0.2321 & 0.3951 & 89.66 & 0.1033 \\
\hline 4 & 0.1562 & 0.3852 & 89.81 & 0.1018 \\
\hline 5 & 0.1219 & 0.4383 & 89.05 & 0.1094 \\
\hline 6 & 0.1120 & 0.3675 & 89.05 & 0.1094 \\
\hline 7 & 0.084 & 0.4057 & 88.75 & 0.1124 \\
\hline 8 & 0.0655 & 0.4074 & 88.75 & 0.1124 \\
\hline 9 & 0.0486 & 0.4150 & 88.44 & 0.1155 \\
\hline 10 & 0.0661 & 0.3712 & 90.12 & 0.0987 \\
\hline
\end{tabular}

\section{CONCLUSION}

Detection of skin cancer lesions as malignant (melanoma) or benign is performed using the CNN RESNET34. The performance of this system is studied using the accuracy and error rate with respect to the variations in number of epochs and learning rate. The accuracy increases with decrease in learning rate. The Maximum accuracy of $90.12 \%$ is achieved when LR is decreased to $1 \times 10-6$ after 10 epochs. In this work, only the detection of skin cancer is considered. In future, it could be extended for the diagnosis of various types of skin cancer such as melanocytic nevi, BCC, SCC through multi class classifier and collecting appropriate data sets. The number of layers in the CNN could also be increased for further improvement in the performance. Strengthening the dataset by increasing the images in each class will also lead better diagnostic results.

\section{REFERENCES}

Apalla, Z., Nashan, D., Weller, R.B. and Castellsagué, X., (2017). Skin cancer: epidemiology, disease burden, pathophysiology, diagnosis, and therapeutic approaches. Dermatology and therapy, 7(1), pp.5-19..

Aswin, R.B., Jaleel, J.A. and Salim, S., (2014). Hybrid genetic algorithm-Artificial neural network classifier for skin cancer detection. In 2014 International Conference on Control, Instrumentation, Communication and Computational Technologies (ICCICCT) (pp. 1304-1309). IEEE..

Miller, D.L. and Weinstock, M.A., (1994). Nonmelanoma skin cancer in the United States: incidence. Journal of the American Academy of Dermatology, 30(5), pp.774778.

Esteva, A., Kuprel, B., Novoa, R.A., Ko, J., Swetter, S.M., Blau, H.M. and Thrun, S., (2017). Dermatologist-level classification of skin cancer with deep neural networks. 
nature, 542(7639), pp.115-118.

He, K., Zhang, X., Ren, S. and Sun, J., (2016). Deep residual learning for image recognition. In Proceedings of the IEEE conference on computer vision and pattern recognition (pp. 770-778).

Hosny, K.M., Kassem, M.A. and Foaud, M.M., (2018). Skin cancer classification using deep learning and transfer learning. In 2018 9th Cairo International Biomedical Engineering Conference (CIBEC) (pp. 90-93). IEEE.

Hosny, K.M., Kassem, M.A. and Foaud, M.M., (2019). Classification of skin lesions using transfer learning and augmentation with Alex-net. PloS one, 14(5), p.e0217293.

https://www.kaggle.com/fanconic/skin-cancermalignant-vs-benign

Jain, S. and Pise, N., (2015). Computer aided melanoma skin cancer detection using image processing. Procedia Computer Science, 48, pp.735-740..

Krizhevsky, A., Sutskever, I. and Hinton, G.E., (2017). Imagenet classification with deep convolutional neural networks. Communications of the ACM, 60(6), pp.8490.

Lau, H.T. and Al-Jumaily, A., (2009), December. Automatically early detection of skin cancer: Study based on neural netwok classification. In 2009 International Conference of Soft Computing and Pattern Recognition (pp. 375-380). IEEE.

Mustafa, S., \&t Kimura, A. (2018, January). An SVM- based diagnosis of melanoma using only useful image features. In 2018 International Workshop on Advanced Image Technology (IWAIT) (pp. 1-4). IEEE.

Mahmoud, M.K.A., Al-Jumaily, A. and Takruri, M., (2011). The automatic identification of melanoma by wavelet and curvelet analysis: study based on neural network classification. In 2011 11th International Conference on Hybrid Intelligent Systems (HIS) (pp. 680-685). IEEE..

Mendes, D.B. and da Silva, N.C., (2018). Skin lesions classification using convolutional neural networks in clinical images. arXiv preprint arXiv:1812.02316..

Rubegni P, Cevenini G, Burroni M, Perotti R, Dell'Eva G, Sbano P, Miracco C, Luzi P, Tosi P, Barbini P, Andreassi L. (2002) Automated diagnosis of pigmented skin lesions. International Journal of Cancer. 2002 0ct 20;101(6): 576-80.

Sasikala, S., Bharathi, M. and Sowmiya, B.R., (2018). Lung Cancer Detection and Classification Using Deep CNN. International Journal of Innovative Technology and Exploring Engineering (IJITEE) ISSN, pp.22783075.

Sasikala, S., Ezhilarasi, M. and Kumar, S.A., (2020). Detection of breast cancer using fusion of MLO and CC view features through a hybrid technique based on binary firefly algorithm and optimum-path forest classifier. In Applied Nature-Inspired Computing: Algorithms and Case Studies (pp. 23-40). Springer, Singapore. 